\title{
Estimation of Food Security Risk Level Using Z-Number-Based Fuzzy System
}

\author{
Rahib H. Abiyev (D), ${ }^{1}$ Kaan Uyar, ${ }^{1}$ Umit Ilhan, ${ }^{1}$ Elbrus Imanov, ${ }^{1}$ and Esmira Abiyeva ${ }^{1,2}$ \\ ${ }^{1}$ Department of Computer Engineering, Applied Artificial Intelligence Research Centre, Near East University, Northern Cyprus, \\ Mersin-10, Turkey \\ ${ }^{2}$ Department of Economics, Near East University, Northern Cyprus, Mersin-10, Turkey
}

Correspondence should be addressed to Rahib H. Abiyev; rahib.abiyev@neu.edu.tr

Received 1 February 2018; Revised 4 April 2018; Accepted 16 April 2018; Published 9 May 2018

Academic Editor: Antonino Malacrinò

Copyright (C) 2018 Rahib H. Abiyev et al. This is an open access article distributed under the Creative Commons Attribution License, which permits unrestricted use, distribution, and reproduction in any medium, provided the original work is properly cited.

\begin{abstract}
Fuzzy logic systems based on If-Then rules are widely used for modelling of the systems characterizing imprecise and uncertain information. These systems are basically based on type- 1 fuzzy sets and allow handling the uncertain and imprecise information to some degree in the developed models. Zadeh extended the concept of fuzzy sets and proposed Z-number characterized by two components, constraint and reliability parameters, which are an ordered pair of fuzzy numbers. Here, the first component is used to represent uncertain information, and the second component is used to evaluate the reliability or the confidence in truth. $Z$ number is an effective approach to solving uncertain problems. In this paper, $Z$-number-based fuzzy system is proposed for estimation of food security risk level. To construct fuzzy If-Then rules, the basic parameters cereal yield, cereal production, and economic growth affecting food security are selected, and the relationship between these input parameters and risk level are determined through If-Then fuzzy rules. The fuzzy interpolative reasoning is proposed for construction of inference mechanism of a $Z$-number-based fuzzy system. The designed system is tested using Turkey cereal data for assessing food security risk level and prediction periods of the food supply.
\end{abstract}

\section{Introduction}

In the real world, the data are often imperfect because of their unreliability and nature. The complementary aspects of imperfect information are uncertainty and imprecision $[1,2]$. Uncertainty characterizes the degree of truth, and imprecision characterizes the content of the data. Fuzzy set theory, introduced by Zadeh [1], can be used to deal with these factors in the modelling of the systems. Therefore, different industrial and nonindustrial problems that were characterized by uncertainty and imprecision were solved by means of the fuzzy set theory [2]. In this paper, the application of fuzzy sets to measurement of food security is considered.

The food security, as it was defined by United Nations' Committee on World Food Security, is a social, physical, and economic access to the sufficient nutritious food that meets the needs of the pupil and also food preferences for an active and healthy life. Food security indicators are built on four pillars: availability, access, utilization, and stability [3]. Food security is highly affected by various factors such as a growing global population, changing the climate, rising food prices, and environmental stressors [4-6]. The possibility of negative actions causes a hazard and increases risk level of food security. The analyses of different factors having influences on food security have been done in different research works. Wu.et al. [6] combined the social, economic, and biophysical factors for estimation of the potential risks of food security. The two indicators, per capita food availability and per capita gross domestic product, were used to estimate the food availability, stability, accessibility, and affordability and to assess the potential future risk of food insecurity. Ehrlich et al. [7] examined two views on the causes of global food insecurity: biophysical and demographic. They concluded that the achieving food-secure future for all humanity is positively influenced by the enhancement of equality, the reduction of the human fertility, 
and improvement of the ecosystem health. Hubbard et al. [5] examined a number of factors that contribute to the food security. Wang et al. [8] designed a prediction model through the combination of the stepwise regression method with BP neural network for forecasting the total food yield. Xiao et al. [9] used BP neural network and considered the development of risk early warning system for food security under the supplied chain environment.

The impacts of factors affecting food security are imprecise, and it is difficult to describe them with certain numbers. For this reason, the fuzzy set theory is a very effective way of estimation of food security risk level. Uncertain availability of nutritious and safe foods is also called food insecurity that can be measured by risk level of food security. Nowadays, the fuzzy set theory is one of the important tools for determination of the risk level of food security and also for risk management [10-16]. Wang et al. [10] considered the combination of the fuzzy set theory and analytical hierarchy process for a risk assessment. Lee [11] evaluated the rate of aggregative risk by fuzzy set theory. Using fuzzy set theory and Dempster-Shafer evidence theory, $\mathrm{Xu}$ et al. [12] proposed a fuzzy evidential grain security warning method for food management. In the research works [10-14], using various factors the design of fuzzy rules is performed for management of the risk. Kadir et al. [15] designed a fuzzy system for assessment of food security risk level. The designed system is based on fuzzy IfThen rules that used three important factors cereal production, cereal yield, and economic growth for the determination of food security risk level. The values of these factors are estimated linguistically using fuzzy values. These fuzzy rules are developed using the knowledge of human experts. In the above research papers, the premise and the consequent parts of the fuzzy If-Then rules are basically designed from numeric data sets or linguistic information using type-1 membership functions. Abiyev et al. [16] designed the type-2 fuzzy system for assessment of food security risk level. Jiang et al. [17] presented the failure mode and effect analysis model based on a fuzzy evidential method for evaluation of the risk of failure modes. Ranking of the risk of the failure model is presented by fusing the occurrence, severity, and detection information and Dempster-Shafer theory.

The fuzzy knowledge-based systems used for decisionmaking are basically based on knowledge, experience, intuition, and assumption of humans. Sometimes, these qualities cannot completely cover all the complexity of the considered real-world problem. The modelling of fuzzy uncertainty inherent to the perception of human experts in evaluating the parameters of input and output variables of food security is very important in modelling the decisionmaking process. The fuzziness and reliability are associated with each other during evaluation of uncertainties related to the fuzzy values of input-output variables. The $Z$-number proposed by Zadeh allows modelling such kind of uncertainty [18]. As we know, the concepts in the human brain for evaluating natural events are vague and imprecise. The boundaries of the parameters low, average, or high level used for evaluating input-output variables cereal yield, cereal production, economic growth, and security level are not exactly defined. Therefore, the assertion that follows from them also becomes vague. The usage of $Z$-numbers allows estimation of input and output relationships using the concept of fuzzy information and partial reliability. Zadeh proposed $Z$-number to represent uncertain information using constraint and reliability information. $Z$-number as represented uses an ordered pair of fuzzy numbers $(A, B)$. Here, $A$ is the fuzzy restriction, and $B$ is the reliability used for valuation of $A$ [18]. $Z$-number allows us to use uncertainty measures to estimate the ambiguity associated with the estimating food risk security. The theoretical background of $Z$-number-based systems has been considered in $[18,19]$. $Z$-number-based fuzzy set using fuzzy constraints and reliability factors describe the human knowledge. Based on the concept of $Z$-number, different research works have been done for solving different problems. Aliev et al. [20] and Kang et al. [21] used $Z$-number to solve multicriteria decision-making (MCDM). Kang et al. [21] solved the decision-making problem by converting $Z$-number to crisp numbers using the approach given in [22]. Xiao et al. [23] converted $Z$-number to type-2 fuzzy set to solve multicriteria decision-making. Here, by calculating the centroid type-2 fuzzy sets, it is converted to the crisp numbers for decisionmaking. Azadeh et al. [24] applied $Z$-number to solve the AHP. The research paper uses the approach described in [22] for problem-solving. Lorkowski et al. [25] considered a fair price approach for decision-making under interval, setvalued, fuzzy, and $Z$-number-based uncertainty. Abiyev et al. [26] proposed a $Z$-number-based interpolative reasoning for control of the dynamic plant. $Z$-numbers are used to solve the problems related to computing with words [27] and decision-making [28] problems. In [29-31], using discrete fuzzy numbers, a new vision of $Z$-numbers is described. An aggregation method is presented for group decision-making problems. In [32], a $Z$-number version of the data envelopment analysis (DEA) model is transformed into possible linear programming and then by applying an alternative $\alpha$-cut approach, a crisp linear programming model is obtained. The proposed model is used for a portfolio selection problem. In [33], a Z-number-based risk-minimization negotiation model is designed for a transmission company and a power purchaser under incomplete information. Here, $Z$-number is used to estimate the uncertainty distribution of the annual electricity transmission, and the benefit and the loss measured by the conditional value at risk is analyzed. Aliev et al. [34] presented an approximate reasoning with $Z$ rules on a basis of linear interpolation for evaluation of the job satisfaction and educational achievement of the students. Wang et al. [35] represented experts' opinions by $Z$-numbers and presented a method based on the Choquet integral for MCDM problems using linguistic $Z$-numbers. Wu et al. [36] used experts' opinions for representing a method for ranking $Z$-numbers. Based on this ranking method, the transformation of $Z$-numbers into basic probability assignments is presented. Two experiments on risk analysis and medical diagnosis illustrate the efficiency of the proposed methodology. In [37], the total utility of $Z$-number is applied to determine the ordering of $Z$-numbers. The approach is used in the application of MCDM under uncertain environments, and it is implemented using Gaussian and triangular 
Z-numbers. Jiang et al. [38] presented a method for ranking generalized fuzzy numbers. Here, the weight of centroid points, the spreads of fuzzy numbers, and degrees of fuzziness are taken into consideration. In this paper, the principles of ranking $Z$-numbers are considered. Using intuitive vectorial centroid, $\mathrm{Ku}$ Khalif et al. [39] presented a hybrid fuzzy MCDM model for $Z$-numbers. The presented model is applied to staff recruitment. Aliev et al. [40] presented the ranking of $Z$-numbers using a human-like fundamental approach. The considered approach is based on two main ideas: optimality degrees of $Z$-numbers and adjusting the obtained degrees using a degree of pessimism. Kang et al. [41] presented the stable strategies analysis based on the utility of $Z$-number to simulate the human's competition in the evolutionary games.

The use of Z-numbers in the solution of different problems needs to use efficient inference mechanism. The interpolative reasoning is proposed by Koczy and Hirota $[42,43]$ for the sparse fuzzy rule base. The method can provide the logical interpretation of modus ponens. The proposed method is based on distance measure and used for approximating fuzzy reasoning [42, 43]. Johanyák et al. [44] showed that different distance measures can be used in the fuzzy sets. But these distance measures do not give full information about the shape of the membership functions. Kozcy et al. [43] mentioned that the Kozcy measure based on $\alpha$-cuts of two fuzzy sets can be used to solve this problem. Using $\alpha$-cuts, Koczy and Hirota proposed a fuzzy interpolative approximate reasoning [43]. Kovács et al. [45] and Hsiao et al. [46] used the interpolative reasoning for the solution of different practical problems. The novelty of this paper is emphasized in the following stages: using $\alpha$-cuts and Koczy and Hirota interpolative reasoning, the design of $Z$ number-based interpolative reasoning mechanism is presented; the development of the $Z$-number-based fuzzy inference system for estimation of food security risk level is proposed.

The paper is organized as follows: Section 2 presents the fuzzy rule interpolation. Section 3 describes the design $Z$ number-based fuzzy inference system. Section 4 presents the application of the developed $Z$-number-based interpolative reasoning mechanism for estimation of food security risk level. Section 5 presents the conclusions.

\section{Fuzzy Rule Interpolation}

Fuzzy sets are the extension of classical sets whose elements have degrees of membership. Let's give definition of fuzzy sets and fuzzy triangular membership functions that will be used in this paper.

Definition 1. Assume that $U=\left\{x_{1}, x_{2}, \ldots, x_{n}\right\}$ is the universe of discourse. A fuzzy set $A$ in $U(A \subset U)$ is defined as a set of ordered pairs $\left\{\left(x_{i}, \mu_{A}\left(x_{i}\right)\right)\right\}$. Here, $x_{i} \in U, \mu_{A}: U \rightarrow[0,1]$ is the membership function of $A$, and $\mu_{A}(x) \in[0,1]$ is the degree of membership of $x$ in $A$ [1].

Definition 2. A triangular fuzzy number $A$ is a fuzzy subset defined by $\left(a_{1}, a_{2}, a_{3}\right)$ triplet on $R$, where the membership function can be determined as

$$
\mu_{A}(x)= \begin{cases}0, & -\infty \leq x \leq a_{1} \\ \frac{x-a_{1}}{a_{2}-a_{1}}, & a_{1} \leq x \leq a_{2} \\ \frac{a_{2}-x}{a_{3}-a_{2}}, & a_{2} \leq x \leq a_{3} \\ 0, & a_{3} \leq x \leq \infty .\end{cases}
$$

The fuzzy triangular membership function is often used for the numeric representation of linguistic terms. In the paper, triangular membership functions are used for the representation of the constraint and reliability of $Z$-number.

Fuzzy rule-based systems include fuzzy sets in antecedent and consequent parts of the rules and define relationships between input and output of the system. Using fuzzy rule base and input data, the fuzzy inference is implemented for making output decision. Different fuzzy reasoning mechanisms are suggested to process uncertain information. These mechanisms are mainly based on the analogy and similarity, compositional inference rule, interpolation, and the concept of distance. The processing capabilities, speed, and complexity of these inference mechanisms are important issues.

This paper considers the inference mechanism based on fuzzy interpolative reasoning proposed by Koczy and Hirota $[42,43]$. The fuzzy interpolative reasoning can efficiently be used for processing sparse rule base and requires the satisfaction of the following conditions: the used fuzzy sets should be continuous, convex, and normal, with bounded support.

The interpolative reasoning is based on distance measure between two fuzzy sets. In the paper, the $\alpha$-cut is used to measure distance between fuzzy sets. Let's consider two fuzzy sets: $A_{1}$ and $A_{2}$. $\alpha$-cut of fuzzy sets $A_{1}$ and $A_{2}$ will be denoted as $A_{1}^{\alpha}$ and $A_{2}^{\alpha}$. We say that fuzzy sets $A_{1}$ is less than $A_{2}$, that is, $A_{1}<A_{2}$, if

$$
\inf \left\{A_{1}^{\alpha}\right\}<\inf \left\{A_{2}^{\alpha}\right\} \text { and } \sup \left\{A_{1}^{\alpha}\right\}<\sup \left\{A_{2}^{\alpha}\right\},
$$

where $\inf \left\{A_{1}^{\alpha}\right\}$ and $\inf \left\{A_{2}^{\alpha}\right\}$ are infimum of $A_{1}$ and $A_{2}$, and $\sup \left\{A_{1}^{\alpha}\right\}$ and $\sup \left\{A_{2}^{\alpha}\right\}$ are supremum of $A_{1}$ and $A_{2}$ (Figure 1).

Let us consider interpolative reasoning mechanism. Consider the fuzzy controller based on single-input and single-output fuzzy rule base. Assume that, in the result of observation, the current input variable $X$ is $A^{*}$. Let us determine the value of output $Y$ of the rule base system corresponding to $A^{*}$. Assume that $A^{*}$ is less than fuzzy sets $A_{1}$ and more than the fuzzy set $A_{2}$, that is, $A_{1}<A^{*}<A_{2}$ and also $B_{1}<B_{2}$. Let us determine the system output for the input $A^{*}$. The problem can be expressed as follows:

$$
\begin{aligned}
& \text { Given that } X \text { is } A^{*} \\
& \text { Rules: if } X \text { is } A_{1} \text {, then } Y \text { is } B_{1} \\
& \text { If } X \text { is } A_{2} \text {, then } Y \text { is } B_{2} \\
& \text { Find conclusion } Y=B^{*} \text { ? }
\end{aligned}
$$


In $[42,43]$ it was shown that

$$
\frac{d\left(A^{*}, A_{1}\right)}{d\left(A^{*}, A_{2}\right)}=\frac{d\left(B^{*}, B_{1}\right)}{d\left(B^{*}, B_{2}\right)},
$$

where $d(*)$ is the distance between two fuzzy sets.

Koczy and Hirota $[42,43]$ determined the final fuzzy sets using distance based on $\alpha$ cuts. Using $\alpha$, cut lower $d^{L}$ and upper $d^{U}$ distances between two fuzzy sets can be calculated as follows:

$$
\begin{aligned}
d_{i j}^{L} & =d^{L}\left(A_{i j}^{\alpha}, X_{j}^{\alpha}\right)=d\left(\inf \left\{A_{i j}^{\alpha}\right\}, \inf \left\{X_{j}^{\alpha}\right\}\right) \\
& =\inf \left\{A_{i j}^{\alpha}\right\}-\inf \left\{X_{j}^{\alpha}\right\}, \\
d_{i j}^{U} & =d^{U}\left(A_{i j}^{\alpha}, X_{j}^{\alpha}\right)=d\left(\sup \left\{A_{i j}^{\alpha}\right\}, \sup \left\{X_{j}^{\alpha}\right\}\right) \\
& =\sup \left\{A_{i j}^{\alpha}\right\}-\sup \left\{X_{j}^{\alpha}\right\}, \\
d_{j}^{L} & =d^{L}\left(B_{j}^{\alpha}, Y_{j}^{\alpha}\right)=d\left(\inf \left\{B_{j}^{\alpha}\right\}, \inf \left\{Y_{j}^{\alpha}\right\}\right) \\
& =\inf \left\{B_{j}^{\alpha}\right\}-\inf \left\{Y_{j}^{\alpha}\right\}, \\
d_{j}^{U} & =d^{U}\left(B_{j}^{\alpha}, Y_{j}^{\alpha}\right)=d\left(\sup \left\{B_{j}^{\alpha}\right\}, \sup \left\{Y_{j}^{\alpha}\right\}\right) \\
& =\sup \left\{B_{j}^{\alpha}\right\}-\sup \left\{Y_{j}^{\alpha}\right\} .
\end{aligned}
$$

In (5), the Hamming or Euclidean formula can be used to measure the distances. Using distance measure, Koczy and Hirota $[42,43]$ proposed interpolated conclusion for the output of $2 \mathrm{k}$ rules.

$$
\begin{aligned}
\inf \left\{B_{j}^{* \alpha}\right\} & =\frac{\sum_{i=1}^{2 k}\left(1 / d^{L}\left(A_{i j}^{\alpha}, A_{i}^{* \alpha}\right)\right) \inf \left\{B_{j}^{\alpha}\right\}}{\sum_{i=1}^{2 k} 1 / d^{L}\left(A_{i j}^{\alpha}, A_{i}^{* \alpha}\right)}, \\
\sup \left\{B_{j}^{* \alpha}\right\} & =\frac{\sum_{i=1}^{2 k}\left(1 / d^{U}\left(A_{i j}^{\alpha}, A_{i}^{* \alpha}\right)\right) \sup \left\{B_{j}^{\alpha}\right\}}{\sum_{i=1}^{2 k} 1 / d^{U}\left(A_{i j}^{\alpha}, A_{i}^{* \alpha}\right)},
\end{aligned}
$$

where $B_{j}^{* \alpha}=\left[\inf \left\{B_{j}^{* \alpha}\right\}, \sup \left\{B_{j}^{* \alpha}\right\}\right]$. In Section 3 , we consider the use of interpolative reasoning for finding the output of the $Z$-number fuzzy system.

\section{Z-Number-Based Fuzzy Inference System}

Definition 3. A $Z$-number is an ordered pair of fuzzy numbers denoted as $Z=(A, B)$, where the first component $A$ is a restriction on the values of fuzzy variable $X$, and the second component $B$ is a measure of reliability for the $A$ component [18].

In Figure 2, the triangular membership functions are used to represent the components of $Z$-number. Here, the $A$ will represent the fuzzy value of the variable, and $B$ will represent the degree of truth or reliability measure or probability of $A$; for example, $X$ is $A$ that is referred to as a possibilitic restriction, that is,

$$
B(X): X \text { is } A \rightarrow \operatorname{Poss}(X=u)=\mu_{A}(x),
$$

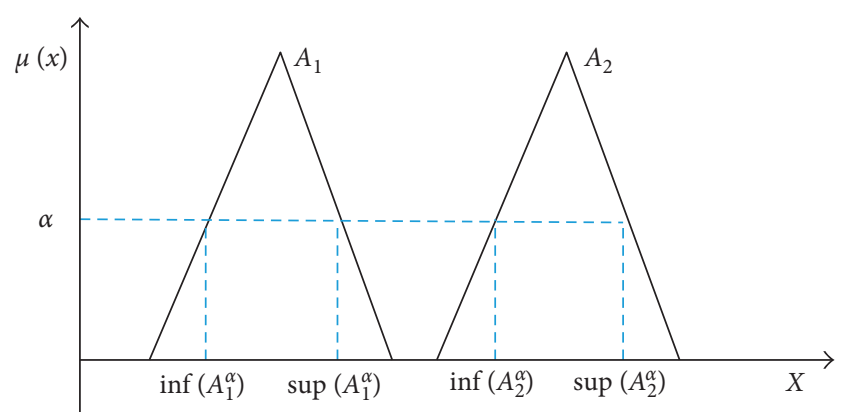

Figure 1: $\alpha$-cut of membership function: infimum and supremum.

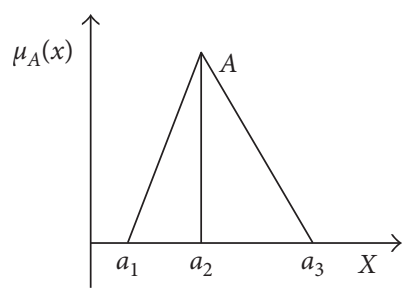

(a)

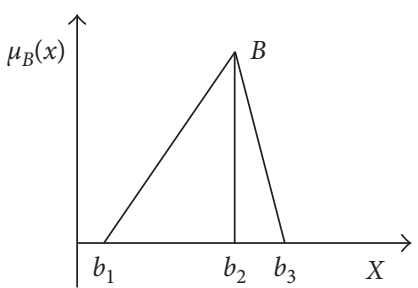

(b)
Figure 2: $Z$-number, $Z=(A, B)$. (a) Restriction; (b) reliability.

where $\mu_{A}(x)$ is membership function of $A$ which may be viewed as constraint-associated with $B(X)$, and $u$ is a generic value of $X . \mu_{A}(x)$ is membership degree to which $u$ satisfies.

$Z$-number is one of an effective way for representation of uncertain information. For example, let us consider the prediction of cereal yield in Turkey. As it is known this parameter depends on number of factors. If we say "This year the cereal yield will be something higher" is considered as a more possible event with the reliability of $100 \%$. This event can be expressed more exactly as "This year the cereal yield will be something higher, very likely."

As shown the event can be described by $Z$-number, that is, " $X$ is $Z=(A, B)$." Here, the fuzzy variable $X$ is "cereal yield." The values of the $X$ variable is described by the pair of $(A, B) . A$ is the constraint which is "something higher," and $B$ is the reliability of $A$ which is "very likely."

Definition 4. Let $Z_{1}=\left(A_{1}, R_{1}\right)$ and $Z_{2}=\left(A_{2}, R_{2}\right)$ be the two $Z$-numbers. The $\alpha$-level distance between $Z$-numbers $Z_{1}$ and $Z_{2}$ is defined as

$$
\begin{aligned}
D\left(Z_{1}^{\alpha}, Z_{2}^{\alpha}\right)= & \left|a_{1}^{L \alpha}-a_{2}^{L \alpha}\right|+\left|a_{1}^{R \alpha}-a_{2}^{R \alpha}\right| \\
& +\left|r_{1}^{L \alpha}-r_{2}^{L \alpha}\right|+\left|r_{1}^{R \alpha}-r_{2}^{R \alpha}\right|,
\end{aligned}
$$

where $a_{1}^{L \alpha}, a_{2}^{L \alpha}, r_{1}^{L \alpha}, r_{2}^{L \alpha}, a_{1}^{R \alpha}, a_{2}^{R \alpha}, r_{1}^{R \alpha}$, and $r_{2}^{R \alpha}$ are $\alpha$-levels of fuzzy sets of left and right sides of fuzzy triangular numbers $A_{1}, R_{1}, A_{2}$, and $R_{2}$ correspondingly [19].

Let us consider the development of $Z$-number-based interpolative reasoning. Assume that multi-input-singleoutput fuzzy If-Then rules have the following form: 


$$
\begin{aligned}
& \text { if } x_{1} \text { is }\left(A_{11}, R_{11}\right), \ldots \text {, and } x_{m} \text { is }\left(A_{1 m}, R_{1 m}\right) \text {, } \\
& \text { then } y \text { is }\left(B_{1}, R_{1}\right), \\
& \text { if } x_{1} \text { is }\left(A_{21}, R_{21}\right), \ldots \text {, and } x_{m} \text { is }\left(A_{2 m}, R_{2 m}\right) \text {, } \\
& \text { then } y \text { is }\left(B_{2}, R_{2}\right), \\
& \text { if } x_{1} \text { is }\left(A_{n 1}, R_{n 1}\right), \ldots \text {, and } x_{m} \text { is }\left(A_{n m}, R_{n m}\right) \text {, } \\
& \text { then } y \text { is }\left(B_{n}, R_{n}\right),
\end{aligned}
$$

where $A_{i j}$ and $R_{i j}$ are fuzzy constraint and reliability parameters, respectively, and $x_{i}$ and $y$ are inputs and output of the system. Here, $j=1, \ldots, m$, and $m$ is a number of input signals; $i=1, \ldots, n$, and $n$ is a number of rules.

Let us use fuzzy interpolation given in above Section 2 for the $Z$-number-based fuzzy rules of Figure 2. Assume those input signals are entered to the input of the fuzzy system. At first iteration, the distance between the incoming input signal and fuzzy values of corresponding variables in the antecedent part of the rules will be computed. Computation of distance is carried out using $\alpha$-cut with the use of the Hamming or Euclidean distances. In the antecedent part, the distances will be calculated for each constraint and reliability variables separately.

Formula (5) is used to find lower and upper distances of membership function in the antecedent part of the rules. $\alpha$ cuts are applied to find the lower and upper distances:

$$
\begin{aligned}
d_{i j}\left(A_{i j}^{\alpha}, X_{j}^{\alpha}\right) & =d_{i j}^{\alpha}=\left|A_{i j}^{\alpha}-X_{j}^{\alpha}\right|, \\
d_{i}^{\alpha} & =\sum_{j}^{m} d_{i j}\left(A_{i j}^{\alpha}, X_{j}^{\alpha}\right),
\end{aligned}
$$

where $d_{j}\left(A_{i j}^{\alpha}, X_{j}^{\alpha}\right)$ is a distance between two fuzzy sets defined for the $i$ th rule; $j=1, \ldots, m$, and $m$ is a number of input signals; $i=1, \ldots, n$, and $n$ is a number of rules. The distances are represented by lower and upper distances, that is, $d_{i j}^{\alpha}\left(A_{i j}^{\alpha}, X_{i}^{\alpha}\right)=$ $\left\{d_{i j}^{L}\left(A_{i j}^{\alpha}, X_{i}^{\alpha}\right), d_{i j}^{U}\left(A_{i j}^{\alpha}, X_{i}^{\alpha}\right\}\right\}$ and $d_{i}^{\alpha}=\left\{d_{i}^{L}, d_{i}^{U}\right\}$. For special case, $\alpha=\{0,1\}$. Formula (10) is applied for constraint $A$ and reliability $R$ values separately. The total distance will be the sum of two distances:

$$
d_{i}^{\alpha}=d c_{i}^{\alpha}+d r_{i}^{\alpha},
$$

where $d c_{i}^{\alpha}$ and $d r_{i}^{\alpha}$ are distances computed by (8) for the constraint and reliability parameters correspondingly. $d_{i}^{\alpha}$ is the distance computed for the ith rule. Each of these distances is characterized by two left and right values:

$$
\begin{aligned}
d c_{i}^{\alpha} & =\left(d c_{i}^{L}, d c_{i}^{U}\right), \\
d r_{i}^{\alpha} & =\left(d r_{i}^{L}, d r_{i}^{U}\right), \\
d_{i}^{\alpha} & =\left(d_{i}^{L}, d_{i}^{U}\right) .
\end{aligned}
$$

The output fuzzy set in the output of the rule is calculated as

$$
\begin{aligned}
Y^{\alpha} & =\frac{\sum_{i=1}^{n}\left(1 / d_{i}^{\alpha}\right) B_{Y_{i}}^{\alpha}}{1 / \sum_{i=1}^{n} 1 / d_{i}^{\alpha}}, \\
R_{Y}^{\alpha} & =\frac{\sum_{i=1}^{n}\left(1 / d_{i}^{\alpha}\right) R_{Y_{i}}^{\alpha}}{1 / \sum_{i=1}^{n} 1 / d_{i}^{\alpha}} .
\end{aligned}
$$

Formula (13) is applied for finding constraint and reliability values of output fuzzy sets. If we combine (10) and (13), then we can get the following equations:

$$
\begin{gathered}
Y^{\alpha}=\frac{\sum_{i=1}^{n}\left(1 / \sum_{j}^{m} d_{L}\left(A_{X_{i, j}}^{\alpha}, X_{j}^{\alpha}\right)\right) B_{Y, i}^{\alpha}}{1 /\left(\sum_{i=1}^{n}\left(1 / \sum_{j}^{m} d_{L}\left(A_{X_{i, j}}^{\alpha}, X_{j}^{\alpha}\right)\right)\right)}, \\
R_{Y}^{\alpha}=\frac{\sum_{i=1}^{n}\left(1 / \sum_{j}^{m} d_{L}\left(A_{X_{i, j}}^{\alpha}, X_{j}^{\alpha}\right)\right) R_{Y, i}^{\alpha}}{1 /\left(\sum_{i=1}^{n}\left(1 / \sum_{j}^{m} d_{L}\left(A_{X_{i, j}}^{\alpha}, X_{j}^{\alpha}\right)\right)\right)} .
\end{gathered}
$$

Using (10)-(13), we can derive the output $Z$ fuzzy signal of the system. It is needed to note that, for finding the output signal, we use $B_{Y i}$, for finding the reliability, we use $R_{Y i}$ variables in the right side of (13) or (14). After getting output signals, conversion of $Z$-number to the crisp number is performed. The formula for calculating the mean of two fuzzy numbers is used for this purpose. The formula $Y=$ $\left(\left(Y_{l}+4 * Y_{m}+Y_{r}\right) / 6\right) *\left(\left(R_{Y_{l}}+4 * R_{Y_{m}}+R_{Y_{r}}\right) / 6\right)$ is used to derive the crisp value of the output signal [21,22]. Here, $Y$ is the fuzzy output value, and $R_{Y}$ is the reliability. It is needed to mention that in the antecedent and consequent parts of the rule base, we are using the triangular type fuzzy sets for the input and output parameters. If we are considering $\alpha=0$ and $\alpha=1$ levels, then we can get left $Y_{l}$, middle $Y_{m}$, and right $Y_{r}$ values of the output signal. Left $\left(Y_{l}, R_{Y_{l}}\right)$ and right $\left(Y_{r}\right.$, $R_{Y_{r}}$ ) values are corresponding to the $\alpha=0$ level, the middle $\left(Y_{m}^{r}, R_{Y_{m}}\right)$ value is corresponding to the $\alpha=1$ level. At first, the left and right values corresponding to the $\alpha=0$ level are determined [26], and then the highest value of the triangle corresponding to the $\alpha=1$ level is determined [46]. These values are used to find output triangular membership function.

\section{Simulation Studies}

The solution of food security risk level starts with the design of appropriate knowledge base. The knowledge base consists of the If-Then rules demonstrating the association between input parameters and output signal. During designing KB, the input and output variables are described by linguistic values. In this paper, $Z$-number-based fuzzy If-Then rules described above are applied for the determination of the food security risk level in Turkey.

The inputs for the system are Turkey cereal yield, Turkey cereal production, and Turkey economic growth. The parameter cereal yield is defined as the monthly farm gate cereal output measured in metric tons, cereal production is defined as cereals that are processed into food products measured in $\mathrm{kg}$ per hectare, and economic growth is defined as the percentage of gross domestic product (GDP) in percentage. The values of the parameters are represented using $Z$-numbers and used for determination of the food security risk level. The decision-making process is conducted with the $Z$-numberbased interpolative inference engine described in Section 3.

The forms of these linguistic values are taken in a triangle form. Each of them is represented by triangle membership functions and has the range. During designing these triangle 


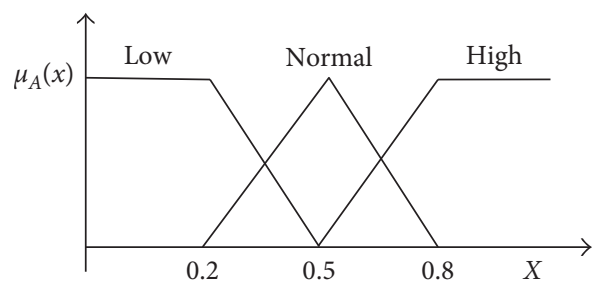

(a)

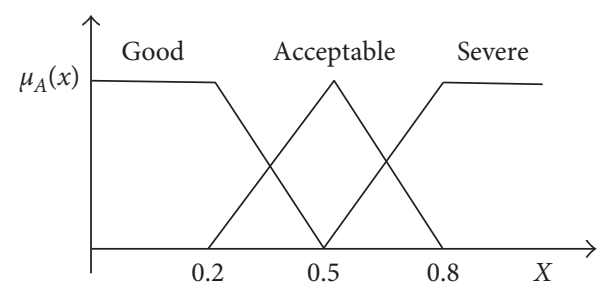

(b)

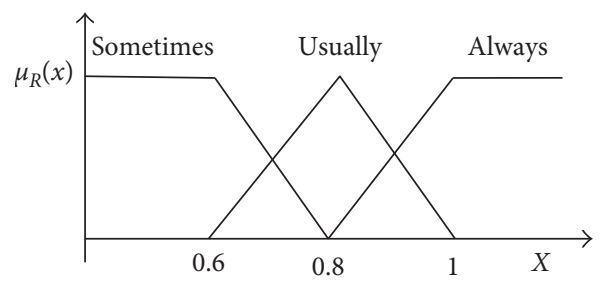

(c)

FIGURE 3: Membership functions used for (a) cereal yield, cereal production, economic growth, (b) risk level, and (c) reliability.

membership functions for the linguistic values, it is necessary to determine the universe of discourse and number of linguistic values for each input parameter. In this paper, the number of membership functions is taken equal to 5 . The linguistic values for the input and output parameters are denoted as NL (negative large), NS (negative small), Z (zero), PS (positive small), and PL (positive large). For simplicity, we scale the range of variables between 0 and 1 . The membership functions used to describe input and output variables are shown in Figure 3.

After defining membership functions for each parameter, the construction of fuzzy If-Then rules is performed. The antecedent part of rule base includes three parameters: cereal yield, cereal production, and economic growth, and the consequent part includes one parameter-security risk level. The construction of the If-Then rules is performed using experts' knowledge. At the same time, the statistical data from the World Bank online database for Turkey are used in construction of rule base. In the design of rule bases, the three linguistic terms are used for each input and output parameters. The values of these linguistic terms are represented using $Z$-numbers. Each fuzzy value is represented by two parameters: constraint and reliability parameters. The membership high yield, normal yield, and low yield functions of constraint and reliability parameters are accepted as triangle. The high yield, normal yield, and low yield linguistic terms are used to represent the cereal yield. The high, normal, and low linguistic terms are used to represent the economic growth parameter. The cereal production is also represented by high, normal, and low linguistic terms. Using the change interval (the ranges) of statistical data, the numeric values of linguistic terms of input parameters are determined. The statistical data are taken from the web page of "Global Food Security Index." Maximum and minimum values of statistical data for the given parameter are used to determine the ranges of input parameters. All the values of parameters are scaled in the interval $0-1$.

The If-Then fuzzy rule base of security risk level is constructed using experts' opinions. The development of rule
TABle 1: Fuzzy knowledge base.

\begin{tabular}{lccc}
\hline Cereal yield & Cereal production & Economic growth & Risk level \\
\hline High & High & High & Good \\
High & High & Normal & Good \\
High & High & Low & Acceptable \\
High & Normal & High & Acceptable \\
High & Normal & Normal & Good \\
High & Normal & Low & Acceptable \\
High & Low & High & Severe \\
High & Low & Normal & Severe \\
High & Low & Low & Severe \\
Normal & High & High & Severe \\
Normal & High & Normal & Acceptable \\
Normal & High & Low & Acceptable \\
Normal & Normal & High & Acceptable \\
Normal & Normal & Normal & Good \\
Normal & Normal & Low & Good \\
Normal & Low & High & Severe \\
Normal & Low & Normal & Acceptable \\
Normal & Low & Low & Severe \\
Low & High & High & Severe \\
Low & High & Normal & Severe \\
Low & High & Low & Severe \\
Low & Normal & High & Severe \\
Low & Normal & Normal & Acceptable \\
Low & Normal & Low & Acceptable \\
Low & Low & High & Severe \\
Low & Low & Normal & Severe \\
Low & Low & Low & Acceptable \\
\hline
\end{tabular}

base is implemented using all possible combination of the values of the input parameters. In the rule base, the values of the parameters are represented using triangular membership functions. After determination of the input fuzzy values, using the opinion of the experts, the construction of the If-Then rules has been performed. The design of If-Then rules is carried out using all possible combination of the values of the input parameters. The input-output associations are defined using expert knowledge. In the rule base, for the input and output constraint and reliability parameters, the values of the 


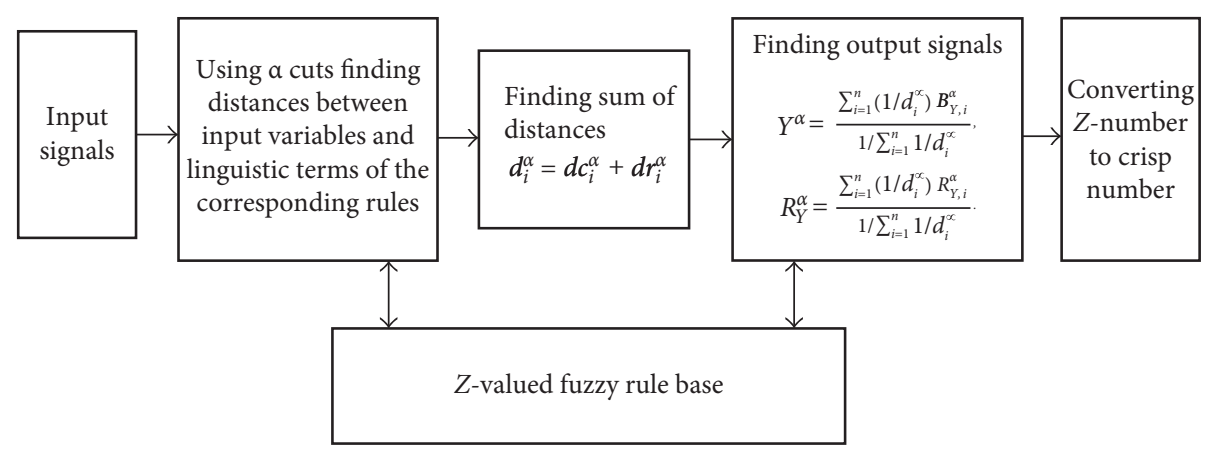

Figure 4: Architecture of the $Z$-number-based system.

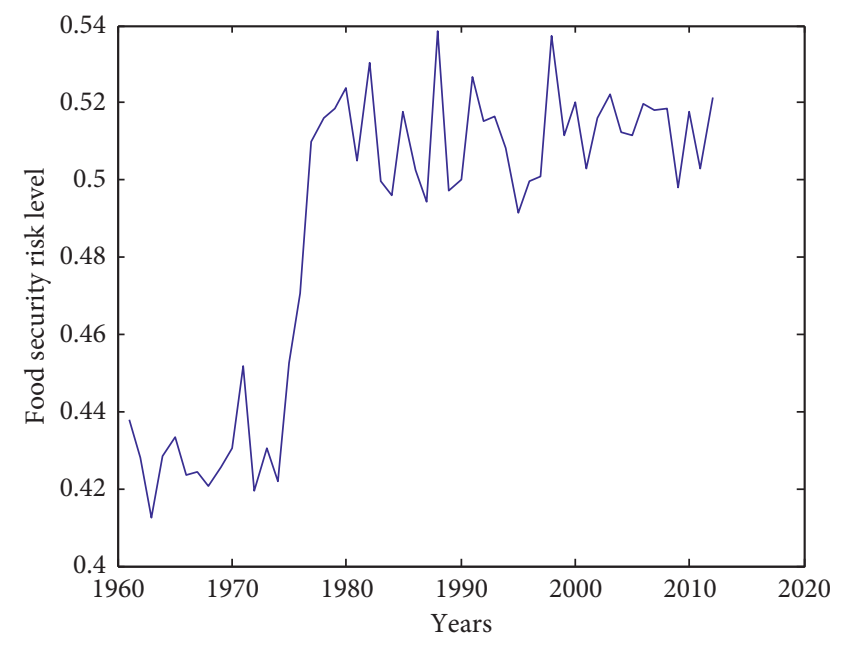

(a)

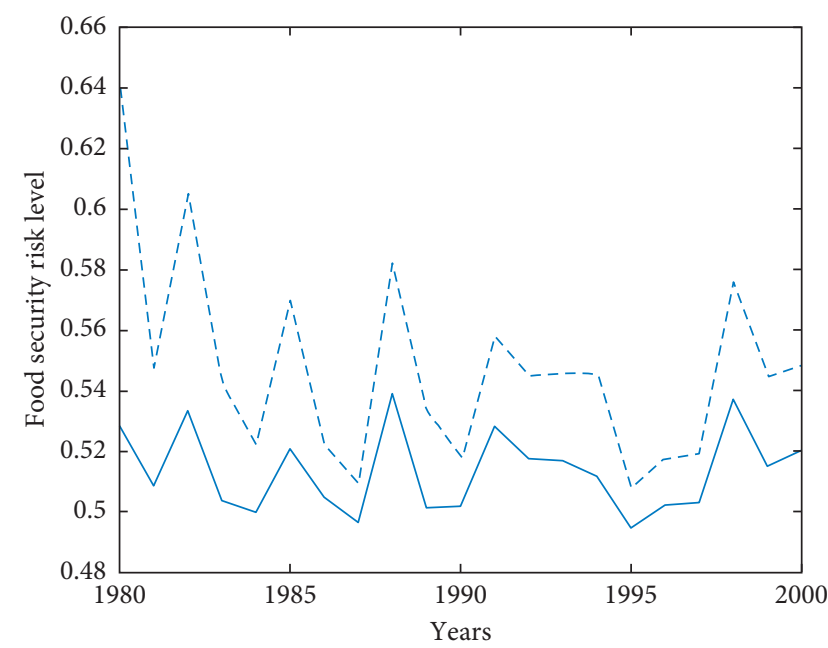

(b)

FIGURE 5: The plots of food security risk level: (a) Z-number-based fuzzy system for the years 1961-2013, (b) comparisons of simulation results of the conventional type-1 fuzzy system (dashed line), and Z-number-based fuzzy system (solid line) for the years 1980-2000.

parameters are represented using triangular interval membership functions. Figure 3 describes the membership functions used for input and output variables.

The output of food security risk level variable is represented using three fuzzy sets: good, acceptable, and severe. The knowledge base representing the input output association is given in Table 1.

The relationship between input and output variables is represented by fuzzy rule base. It was found that high cereal yield and high cereal production lead to the high level of food security. It can be observed that even economic growth is low. But if the quantities of cereal yield and cereal production are low, there may not be enough food.

After the development of fuzzy rule base, the design of the fuzzy inference system is implemented using interpolative reasoning mechanism. The designed system will use fuzzy rule base and statistical input data and make a conclusion about risk level. Using fuzzy rule interpolation, the structure of the inference system is designed (Figure 4). In the structure, the distances between incoming input signals and the membership functions of the antecedent part of If-Then rules are determined. Here, using $\alpha$ cuts distances between input variables, and corresponding linguistic terms of the rules are determined. The same procedure is repeated for reliability parameters also. After this operation, sum of distances are determined. Using sum of distances and active rules based on (13), the fuzzy outputs are determined. For each constraint and reliability parameters, the outputs are determined. Based on the mean formula of two fuzzy numbers, the crisp output of the system is determined.

Using real annual historical data between 1961 and 2013 years in Turkey [47] and the $Z$-number-based fuzzy system, the risk level of food security is determined. The real data are fed into the system input. Figure 5(a) depicts the changes of food security risk level obtained by the $Z$-number-based fuzzy system for the statistical data between 1961 and 2013 year. As shown every year, the assessment of risk level of food security is changed according to the change of the values of cereal yield, cereal production, and economic growth. The risk level of food security is acceptable between 0 and 0.8. As an example, if we consider 1988 and 1998 years, then we can observe that when Turkey cereal yield was $1742.108 \mathrm{~kg} / \mathrm{ha}$, cereal production was 23498600 tons, and economic growth was $0.29 \%$, the value of food security risk level was 0.54 for 1988 . When cereal yield was $2075.197 \mathrm{~kg} / \mathrm{ha}$, cereal production was 28885720 tons, and economic growth 
was $-3.36 \%$, the value of food security risk level was 0.535 for 1998. Both of them are related to the food security risk level conditions "Acceptable" with the membership $\approx 0.87$.

The system demonstrates that the high values of cereal yield and cereal production will lead to the high security level if economic growth is low or normal. In these cases, most people will pay low prices for their food, and consumer will expend fewer resources to get the best food; then, in this situation, the expensive high-quality food may be wasted. But when economic growth is high and cereal yield and cereal production are low, food security will be low and there may not be enough food for everyone.

In the second simulation, for comparison purpose, using the rule base given in Table 1, the design of a conventional type-1 fuzzy system with the interpolative reasoning is carried out. For clear comparison, we plotted the changes in the risk levels for the conventional type- 1 fuzzy systems and $Z$-number-based system for the years 1980-2000 that is given in Figure 5(b). Here, the solid line is the security risk level obtained by the $Z$-number-based system, and the dashed line is the security risk level obtained by the conventional type-1 fuzzy system. As shown, there are some differences between the simulation results. If we consider for the years 1988 and 1998, the values of food security risk level for the conventional type-1 fuzzy system were obtained as 0.62 and 0.56 correspondingly. The results are between the conditions of "Acceptable" and "Severe." The first result with the membership 0.6 is related to the condition of "Acceptable," and the membership 0.4 is related to the condition of "Severe." The second one with the membership 0.8 is related to the condition of "Acceptable," and the membership 0.2 is related to the condition "Severe." As shown from the graphics, the reliability degrees for the linguistic values in rule base affect the food security risk level. If we try to get the Znumber-based system output using reliability degree of each linguistic value equal to one, then we can get the same results for the food security risk level as in the conventional type-1 fuzzy system. True assessment shows that the value of reliability degree allows getting more accurate value for the food security risk level.

From the simulation, it was obvious that the food security risk level is acceptable in all the time period for both systems. As shown from the figure, the results given by the $Z$-numberbased fuzzy system is more reliable than another one.

\section{Conclusions}

In this paper, the design of $Z$-number-based fuzzy system is implemented for determining food security risk level. Based on Koczy-Hirota interpolative reasoning, the $Z$-numberbased inference engine is designed. The designed system is applied for measuring the food security risk level in Turkey. Using input parameters cereal yield, cereal production, and economic growth, the output food security risk level is evaluated and the rule base is developed. The relationships between input and output variables are expressed using $Z$ valued numbers. The designed system is tested using statistical data taken from the Global Security Index database for 1961 and 2013. Based on the $Z$-number-based inference system, rule base and statistical data, the prediction of the risk level of food security of Turkey is performed. The obtained results demonstrate the applicability of the designed system in real life.

\section{Data Availability}

The data used to support the findings of this study are available from the corresponding author upon request.

\section{Conflicts of Interest}

The authors declare that there are no conflicts of interest regarding the publication of this paper.

\section{References}

[1] L. A. Zadeh, "Fuzzy sets," Information and Control, vol. 8, no. 3, pp. 338-353, 1965.

[2] J. Yen, R. Langari, and L. A. Zadeh, Industrial Applications of Fuzzy Logic and Intelligent Systems, IEEE Press Piscataway, Hoboken, NJ, USA, 1995.

[3] FAO 2006, Food Security: Policy Brief, 2006, http://www.fao. org/forestry/13128-0e6f36f27e0091055bec28ebe830f46b3.pdf.

[4] C. Peihong and W. Jiaqiong, "Application of a fuzzy AHP method to risk assessment of international construction projects," in Proceedings of International Conference on Electronic Commerce and Business Intelligence, pp. 459-462, Beijing, China, June 2009.

[5] L. J. Hubbard and C. Hubbard, "Food security in the United Kingdom: external supply risks," Food Policy, vol. 43, pp. 142-147, 2013.

[6] W. Wu, P. Yang, H. Tang, O. Zhou, Z. Chen, and R. Shibasaki, "A method for spatially explicit assessment of potential risks of food insecurity," Agriculture and Agricultural Science Procedia, vol. 1, pp. 325-333, 2010.

[7] P. R. Ehrlich and J. Harte, "Food security requires a new revolution," International Journal of Environmental Studies, vol. 72, no. 6, pp. 908-920, 2015.

[8] G. Z. Wang, L. Zhang, and L. Lu, "The prediction model of food security based on stepwise regression method and bp neural network," in International Conference on Environmental Science and Information Application Technology, pp. 1214-1217, Wuhan, China, July 2009.

[9] J. Xiao, Z. S. Ma, and D. J. Zhang, "The research on the BP neural network in the food security risk early warning applied under supply chain environment," in Proceedings of the Second International Symposium on Knowledge Acquisition and Modeling, vol. 2, pp. 281-284, Wuhan, China, November 2009.

[10] X. Wang, D. Li, and X. Shi, "A fuzzy enabled model for aggregative food safety risk assessment in food supply chains," in Proceedings of IEEE International Conference on Service Operations and Logistics, and Informatics, vol. 2, pp. 28982903, Beijing, China, October 2008.

[11] H. M. Lee, "Applying fuzzy set theory to evaluate the rate of aggregative risk in software development," Fuzzy Sets and Systems, vol. 79, no. 3, pp. 323-36, 1996.

[12] J. Xu and Y. Deng, "Linguistic ranking model and its application in food management," in Proceedings of International Conference on Computer Design and Applications, vol. 5, pp. 208-212, Qinhuangdao, China, June 2010. 
[13] Q. Yu, W. Wu, H. Tang, Y. Chen, and P. Yang, "Notice of retraction. Evaluating food security in APEC region based on grain productivity," in Proceedings of Second IITA International Conference on Geoscience and Remote Sensing, pp. 463-467, Qingdao, China, August 2010.

[14] N. Perrot, I. Ioannou, I. Allais, C. Curt, J. Hossenlopp, and G. Trystram, "Fuzzy concepts applied to food product quality control: a review," Fuzzy Sets and Systems, vol. 157, no. 9, pp. 1145-1154, 2006.

[15] M. K. A. Kadir, E. I. Hines, K. Qaddoum et al., "Food security risk level assessment: a fuzzy logic-based approach," Applied Artificial Intelligence: An International Journal, vol. 27, no. 1, pp. 50-61, 2013.

[16] R. H. Abiyev, K. Uyar, U. Ilhan, and E. Imanov, "Assessment of food security risk level using type 2 fuzzy system," Procedia Computer Science, vol. 102, pp. 547-554, 2016.

[17] W. Jiang, C. Xie, M. Zhuang, and Y. Tang, "Failure mode and effects analysis based on a novel fuzzy evidential method," Applied Soft Computing, vol. 57, pp. 672-683, 2017.

[18] L. A. Zadeh, "A note on a Z-number," Information Science, vol. 181, no. 14, pp. 2923-2932, 2011.

[19] R. A. Aliev, O. H. Huseynov, R. R. Aliyev, and A. V. Alizadeh, The Arithmetic on Z-Numbers. Theory and Application, World Scientific Publishing Co., Singapore, 2015.

[20] R. A. Aliev and L. M. Zeinalova, "Decision making under Z-information," in Human-Centric Decision-Making Models for Social Sciences (Studies in Computational Intelligence), P. Guo and W. Pedrycz, Eds., pp. 233-252, Springer, Berlin, Germany, 2014.

[21] B. Kang, D. Wei, Y. Li, and Y. Deng, "Decision making using z-numbers under uncertain environment," Journal of Computational Information Systems, vol. 8, no. 7, pp. 2807-2814, 2012.

[22] B. Kang, D. Wei, Y. Li, and Y. Deng, "A method of converting z-number to classical fuzzy number," Journal of Computational Information System, vol. 9, no. 3, pp. 703-709, 2012.

[23] Z. Q. Xiao, "Application of Z-numbers in multi-criteria decision making," in Proceedings of the Informative and International Conference on Cybernetics for Computational Social Systems, pp. 91-95, Qingdao, China, 2014.

[24] A. Azadeh, M. Saberi, N. Z. Atashbar, E. Chang, and P. Pazhoheshfar, "Z-AHP: a Z-number extension of fuzzy analytical hierarchy process," in Proceedings of International Conference on Digital Ecosystems and Technologies (DEST), pp. 141-147, Menlo Park, CA, USA, July 2013.

[25] J. Lorkowski, V. Kreinovich, and R. A. Aliev, "Towards decision making under interval, set-valued, fuzzy, and z-number uncertainty: a fair price approach," in Proceedings of IEEE International Conference on Fuzzy Systems, Beijing, China, July 2014.

[26] R. H. Abiyev, "Z-number based fuzzy inference system for dynamic plant control," Advances in Fuzzy Systems, vol. 2016, Article ID 8950582, 7 pages, 2006.

[27] S. K. Pal, R. Banerjee, S. Dutta, and S. S. Sarma, "An insight into the Z-number approach to CWW," Fundamenta Informaticae, vol. 124, no. 1-2, pp. 197-229, 2013.

[28] R. A. Aliev, W. Pedrycz, A. V. Alizadeh, and O. H. Huseynov, "Fuzzy optimality based decision making under imperfect information without utility," Fuzzy Optimization and Decision Making, vol. 12, no. 4, pp. 357-372, 2013.

[29] R. R. Yager, "On Z-valuations using Zadeh's Z-numbers," International Journal of Intelligent Systems, vol. 27, no. 3, pp. 259-278, 2012.

[30] S. T. Massane, J. V. Riera, and J. Torrens, "A new vision of Zadeh's Z-numbers," in Proceedings of the International
Conference on Information Processing and Management of Uncertainty in Knowledge-Based Systems, pp. 581-592, Eindhoven, Netherlands, June 2016.

[31] S. T. Massane, J. V. Riera, and J. Torrens, "On the aggregation of Zadeh's Z-numbers based on discrete fuzzy numbers," in Advances in Intelligent Systems and Computing, V. Torra, R. Mesiar, and B. Baets, Eds., pp. 118-129, Springer, Berlin, Germany, 2018.

[32] A. Azadeh, R. Kokabi, and D. E. A. Znumber, "A new possibilistic DEA in the context of Z-numbers," Advanced Engineering Informatics, vol. 30, no. 3, pp. 604-617, 2016.

[33] B. Zou, Y. Zhou, J. Hu et al., "Z-number-based negotiation model for determining two-part transmission tariffs of crossregional transmission projects," IET Generation, Transmission and Distribution, vol. 11, no. 7, pp. 1829-1838, 2017.

[34] R. A. Aliev, W. Pedrycz, O. H. Huseynov, and S. Z. Eyupoglu, "Approximate reasoning on a basis of Z-number valued IfThen rules," IEEE Transactions on Fuzzy Systems, vol. 25, no. 6, pp. 1589-1600, 2017.

[35] J. Q. Wang, Y. X. Cao, and H. Y. Zhang, "Multi-criteria decision-making method based on distance measure and Choquet integral for linguistic Z-numbers," Cognitive Computation, vol. 9, no. 6, pp. 827-842, 2017.

[36] D. Wu, X. Liu, F. Xue, H. Zheng, Y. Shou, and W. Jiang, "A new medical diagnosis method based on Z-numbers," Applied Intelligence, vol. 48, no. 4, pp. 854-867, 2017.

[37] B. Kang, Y. Deng, and R. Sadiq, "Total utility of Z-number," Applied Intelligence, vol. 48, no. 3, pp. 703-729, 2017.

[38] W. Jiang, C. Xie, Y. Luo, and Y. Tang, "Ranking Z-numbers with an improved ranking method for generalized fuzzy numbers," Journal of Intelligent and Fuzzy Systems, vol. 32, no. 3, pp. 1931-1943, 2017.

[39] K. M. N. Ku Khalif, A. Gegov, and A. S. Abu Bakar, "Hybrid fuzzy MCDM model for Z-numbers using intuitive vectorial centroid," Journal of Intelligent and Fuzzy Systems, vol. 33, no. 2, pp. 791-805, 2017.

[40] R. A. Aliev, O. H. Huseynov, and R. Serdaroglu, "Ranking of Z-numbers and its application in decision making," International Journal of Information Technology and Decision Making, vol. 15, no. 6, pp. 1503-1519, 2015.

[41] B. Kang, G. ChhipiShrestha, Y. Deng et al., "Stable strategies analysis based on the utility of Z-number in the evolutionary games," Applied Mathematics and Computation, vol. 324, pp. 202-217, 2018.

[42] L. T. Koczy and K. Hirota, "Approximate reasoning by linear rule interpolation and general approximation," International Journal of Approximate Reasoning, vol. 9, no. 3, pp. 197-225, 1993.

[43] L. T. Koczy and K. Hirota, "Interpolative reasoning with insufficient evidence in sparse fuzzy rule bases," Information Sciences, vol. 71, no. 1, pp. 169-201, 1993.

[44] Z. C. Johanyák and S. Kovács, "Distance based similarity measures of fuzzy sets," in Proceedings of the 3rd SlovakianHungarian Joint Symposium on Applied Machine Intelligence, pp. 265-276, Herlany, Slovakia, January 2005.

[45] S. Kovács and L. T. Kóczy, "Application of interpolationbased fuzzy logic reasoning in behaviour-based control structures," in Proceedings of the IEEE International Conference on Fuzzy Systems, pp. 25-29, Budapest, Hungary, July 2004.

[46] W. H. Hsiao, S. M. Chen, and C. H. Lee, "A new interpolative reasoning method in sparse rule-based systems," Fuzzy Sets and Systems, vol. 95, no. 1, pp. 17-22, 1998.

[47] World Databank, Explore. Create, Share: Development Data, 2015, http://databank.worldbank.org/ddp/home.do. 


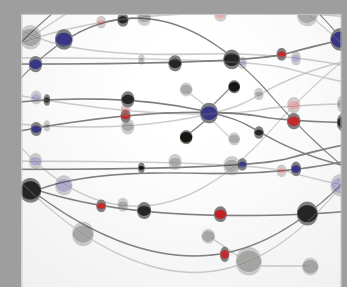

The Scientific World Journal
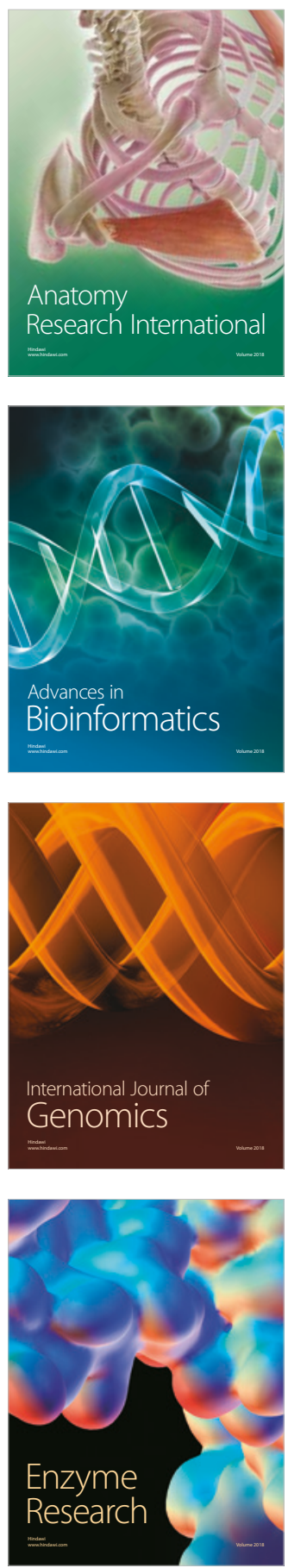
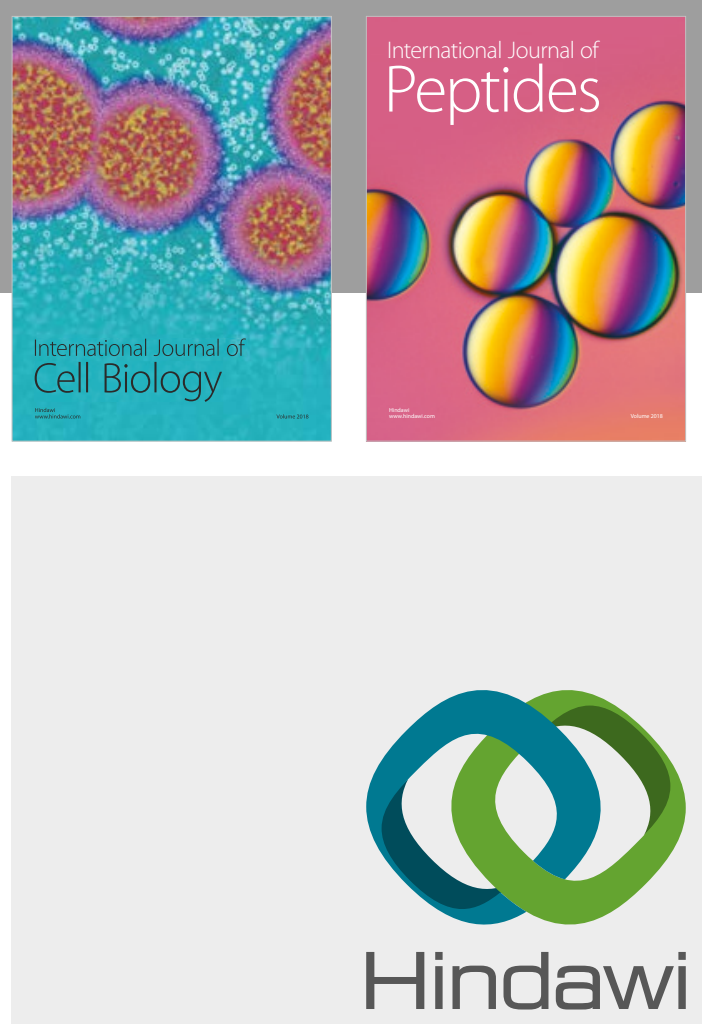

Submit your manuscripts at

www.hindawi.com
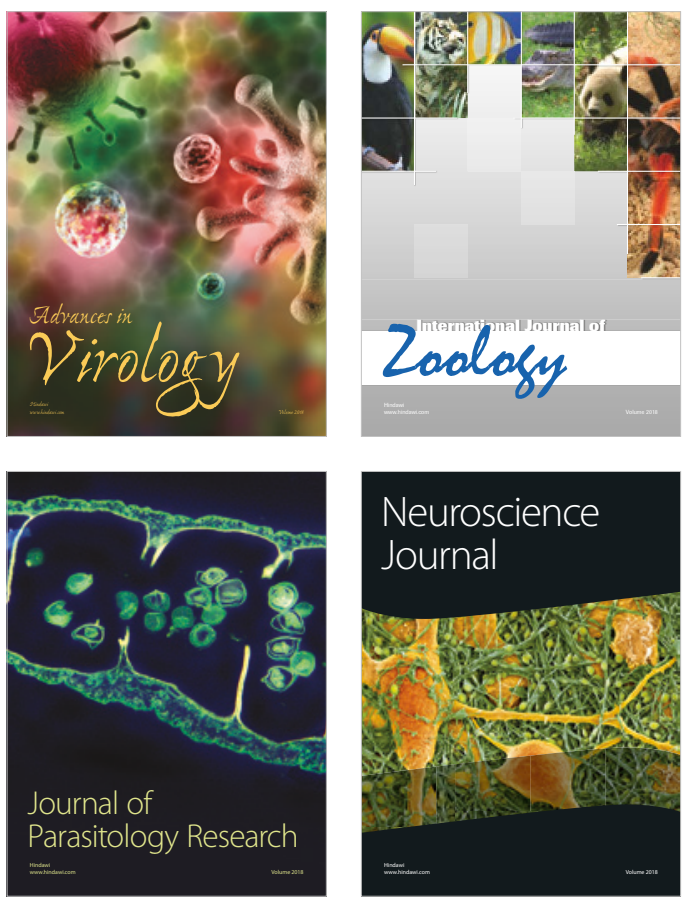
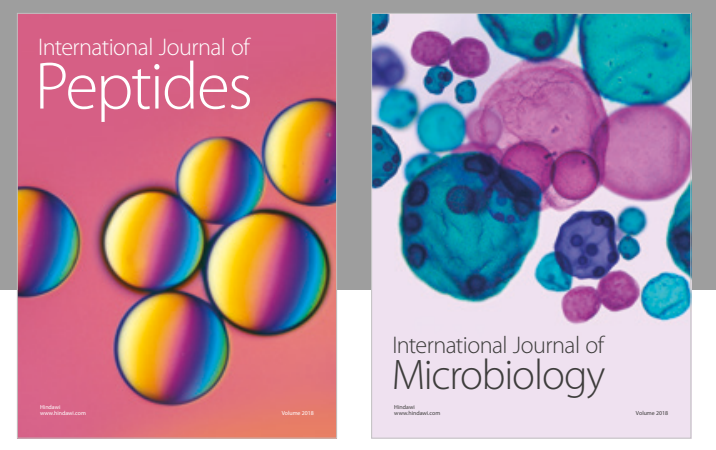

nternational Journal of Microbiology
Journal of
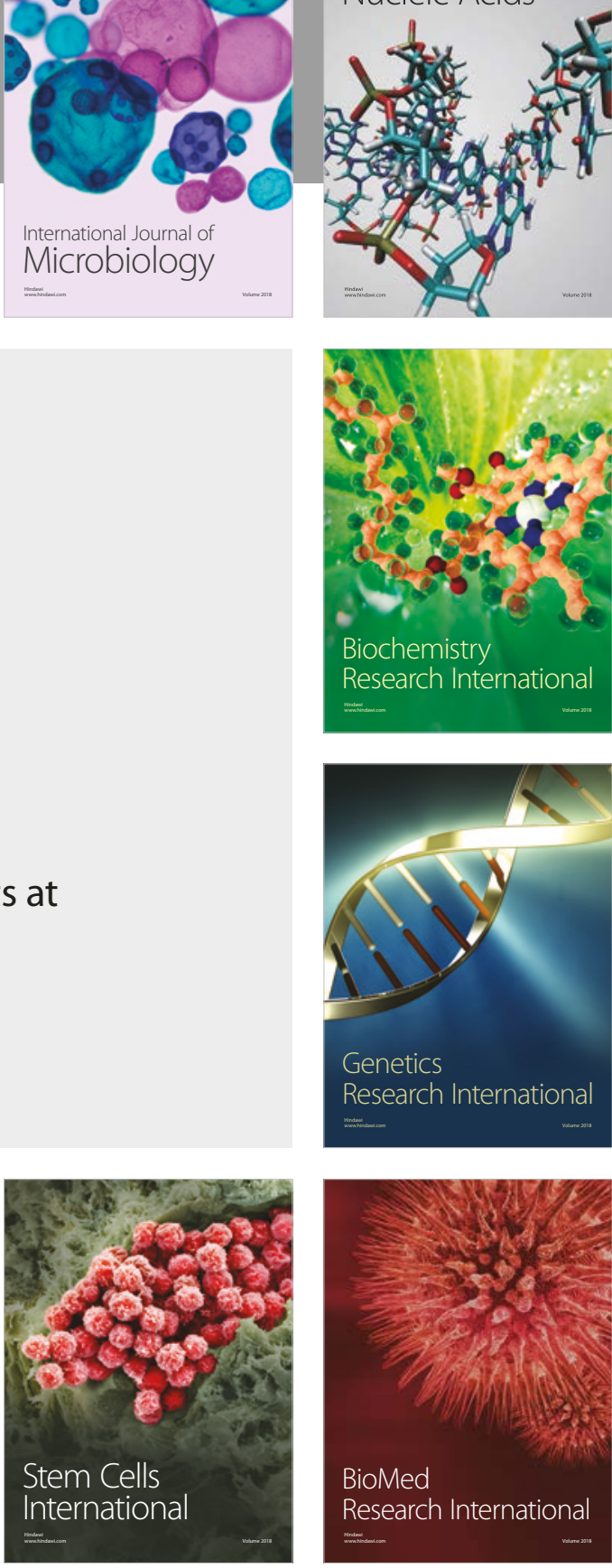
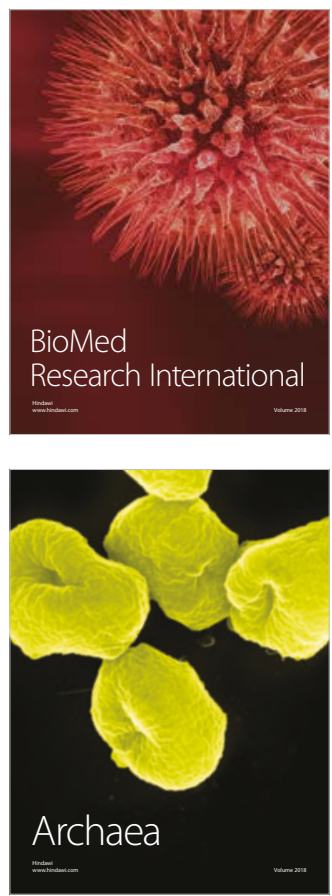